\title{
Caractérisation De La Diversité, De La Structure Des Tailles Et Du Stress Écologique Au Niveau Du Peuplement Des Poissons Sur Le Fleuve Bandama (Côte d'Ivoire, Afrique De l'Ouest)
}

\author{
Kien Kouassi Brahiman, \\ Ndiaye Awa, \\ UFR Sciences Biologiques, Université Peleforo Gon Coulibaly, Korhogo, \\ Côte d'Ivoire. \\ Aboua Benié Rose Danielle, \\ Laboratoire des Milieux Naturels et Conservation de la Biodiversité, UFR \\ Biosciences, Université Félix-Houphouët-Boigny, Abidjan, Côte d’Ivoire. \\ Doi:10.19044/esj.2021.v17n43p260
}

Submitted: 20 September 2021

Accepted: 28 December 2021

Published: 31 December 2021
Copyright 2021 Author(s)

Under Creative Commons BY-NC-ND 4.0 OPEN ACCESS

Cite As:

Brahiman K.K.,Awa N., \& Danielle A.B.R.,(2021). Caractérisation De La Diversité, De La Structure Des Tailles Et Du Stress Écologique Au Niveau Du Peuplement Des Poissons Sur Le Fleuve Bandama (Côte d'Ivoire, Afrique De l'Ouest) European Scientific Journal, ESJ, 17(43), 260.

https://doi.org/10.19044/esj.2021.v17n43p260

\section{Résumé}

La connaissance de la faune ichtyologique des eaux ivoiriennes préoccupe à la fois les scientifiques et les responsables de développement du secteur de la pêche. En effet, l'intensification croissante de l'exploitation des ressources halieutiques en milieu continental fait planer de réels risques de régression et de disparition des espèces.

Cette étude vise à caractériser la variation spatio-temporelle de la faune ichtyologique et d'identifier les indices biologiques qui déterminent la distribution des espèces de poissons et l'état d'exploitation de ces poissons dans le fleuve Bandama.

Les poissons ont été échantillonnés de juillet 2019 à juin 2020 à partir des captures des pêcheurs locaux. Chaque spécimen identifié, est pesé et mesuré.

L'analyse spatio-temporelle a indiqué une variation non significative $(p>0,05)$ de la faune ichtyologique et des indices de diversité $\mathrm{H}^{\prime}$ et $\mathrm{E}$. Néanmoins, L'analyse de la structure du peuplement (H' de 2,09 à 2,34; E de 
0,83 à 0,85 ) a révélé un milieu fluvial assez diversifié. Il est dominé par les Cichlidae et Cyprinidae au niveau des familles et Labeo coubie, Coptodon zillii et Chrysichthys nigrodigitatus au niveau des espèces quel que soit le site de pêche. Ce peuplement est composé majoritairement de spécimens juvéniles. Les résultats de ce travail constituent une étude de base pour les gouvernants dans la mise en place de plan de gestion durable des ressources halieutiques en milieu continental.

L'essentiel de la production du fleuve Bandama est fourni par les Cichlidae et dans la localité de Tiassalé. Néanmoins, les indices de diversité calculés montrent une ichtyofaune diversifiée. Cette ichtyofaune est dominée par des juvéniles.

Mots clés: Ichtyofaune, Diversité, Tailles, Fleuve, Bandama, Côte d’Ivoire.

\title{
Characterization of the Diversity, Size Structure and Ecological Stress in Fish Populations on the Bandama River (Ivory Coast, West Africa)
}

\author{
Kien Kouassi Brahiman, \\ Ndiaye Awa, \\ UFR Sciences Biologiques, Université Peleforo Gon Coulibaly, Korhogo, \\ Côte d'Ivoire. \\ Aboua Benié Rose Danielle, \\ Laboratoire des Milieux Naturels et Conservation de la Biodiversité, UFR \\ Biosciences, Université Félix-Houphouët-Boigny, Abidjan, Côte d’Ivoire
}

\begin{abstract}
Knowledge of the ichthyological fauna of Ivorian waters is of concern to both scientists and those responsible for the development of the fishing sector. In fact, the increasing intensification of the exploitation of fishery resources in the continental environment poses real risks of regression and disappearance of species.

This study aims to characterize the spatio-temporal variation of the ichthyological fauna and to identify the biological indices which determine the distribution of fish species and the state of exploitation of these fish in the Bandama River.

The fish were sampled from July 2019 to June 2020 from the catches of local fishermen. Each identified specimen is weighed and measured.

The spatio-temporal analysis indicated a non-significant variation ( $>$ 0.05 ) in the ichthyological fauna and the indices of diversity $\mathrm{H}$ 'and $\mathrm{E}$. Nevertheless, the analysis of the structure of the stand ( $\mathrm{H}$ ' of 2.09 at 2.34; E
\end{abstract}


from 0.83 to 0.85 ) revealed a fairly diverse fluvial environment. It is dominated by Cichlidae and Cyprinidae at family level and Labeo coubie, Coptodon zillii and Chrysichthys nigrodigitatus at species level regardless of the fishing site. This stand is mainly composed of juvenile specimens. The results of this work constitute a basic study for those in charge in the establishment of a plan for the sustainable management of fishery resources in the continental environment.

Most of the production of the Bandama River is supplied by the Cichlidae and in the locality of Tiassalé. Nevertheless, the calculated diversity indices show a diversified ichthyofauna. This ichthyofauna is dominated by juveniles.

Keywords: Ichthyofauna, Diversity, Sizes, Bandama River, Côte d’Ivoire

\section{Introduction}

La connaissance de la faune ichtyologique des rivières et des plans d'eau africains préoccupe à la fois les scientifiques et les responsables du développement car l'intensification croissante de l'exploitation des ressources halieutiques en milieu continental en Afrique associée aux processus de dégradation du milieu naturel font planer de réels risques de régression et de disparition des espèces (Lalèyè et al., 2004). Ainsi, Monchowui et al. (2008) proposent des mesures de conservation et de gestion rationnelle des stocks des peuplements de poissons. Ces mesures prennent en compte l'écologie, la composition et le niveau d'exploitation de la faune ichtyologique des plans d'eau. Toute mesure de conservation de la faune ichtyologique passe nécessairement par une bonne connaissance des espèces de poissons, des indices qui caractérisent leur distribution (Wu et al. 2011 ; Kamelan et al., 2013) et leur niveau d'exploitation (Kantoussan, 2007).

Le manque d'information sur ces différents aspects constitue un handicap dans l'élaboration et la conduite de plans d'aménagement et de gestion pour la protection de la faune ichtyologique.

En Côte d'Ivoire, les études de connaissance de la faune ichtyologique ont été menées sur les lacs de barrages hydroélectriques (Da Costa et Konan, 2005 ; Da Costa et Dietoa, 2007 ; Tah et al., 2009 ; Koudou, 2012) et les bassins versants des rivières et des fleuves. Sur le fleuve Bandama, l'étude réalisée par Aboua (2012) a mis en relief uniquement l'inventaire de l'ichtyofaune et la mise en place d'un indice d'intégrité biotique. Le présent travail réalisé sur le fleuve Bandama est un complément à cette étude pour la constitution d'une base de données sur l'activité halieutique pratiquée sur les bassins versants des fleuves et rivières de Côte d'Ivoire. Cette étude a donc été initiée dans le but d'approfondir les connaissances sur la caractérisation de la diversité spécifique du peuplement ichtyologique du fleuve Bandama. Le présent travail 
s’attèle à décrire la répartition spatio-temporelle de la faune ichtyologique et les indices biologiques qui la caractérisent d'une part et d'autre part la pression de la pêche sur ce peuplement du fleuve Bandama.

\section{Matériel et méthodes \\ Milieu d'étude}

La présente étude a été réalisée au niveau de trois sites sur le cours inférieur du fleuve Bandama (Figure 1): Singrobo ( $6^{\circ} 05 \mathrm{~N}$ et $4^{\circ} 55 \mathrm{O}$ ), N’Zianouan $\left(6^{\circ} 00 \mathrm{~N}\right.$ et $\left.4^{\circ} 49 \mathrm{O}\right)$ et Tiassalé $\left(5^{\circ} 53 \mathrm{~N}\right.$ et $\left.4^{\circ} 49 \mathrm{O}\right)$.

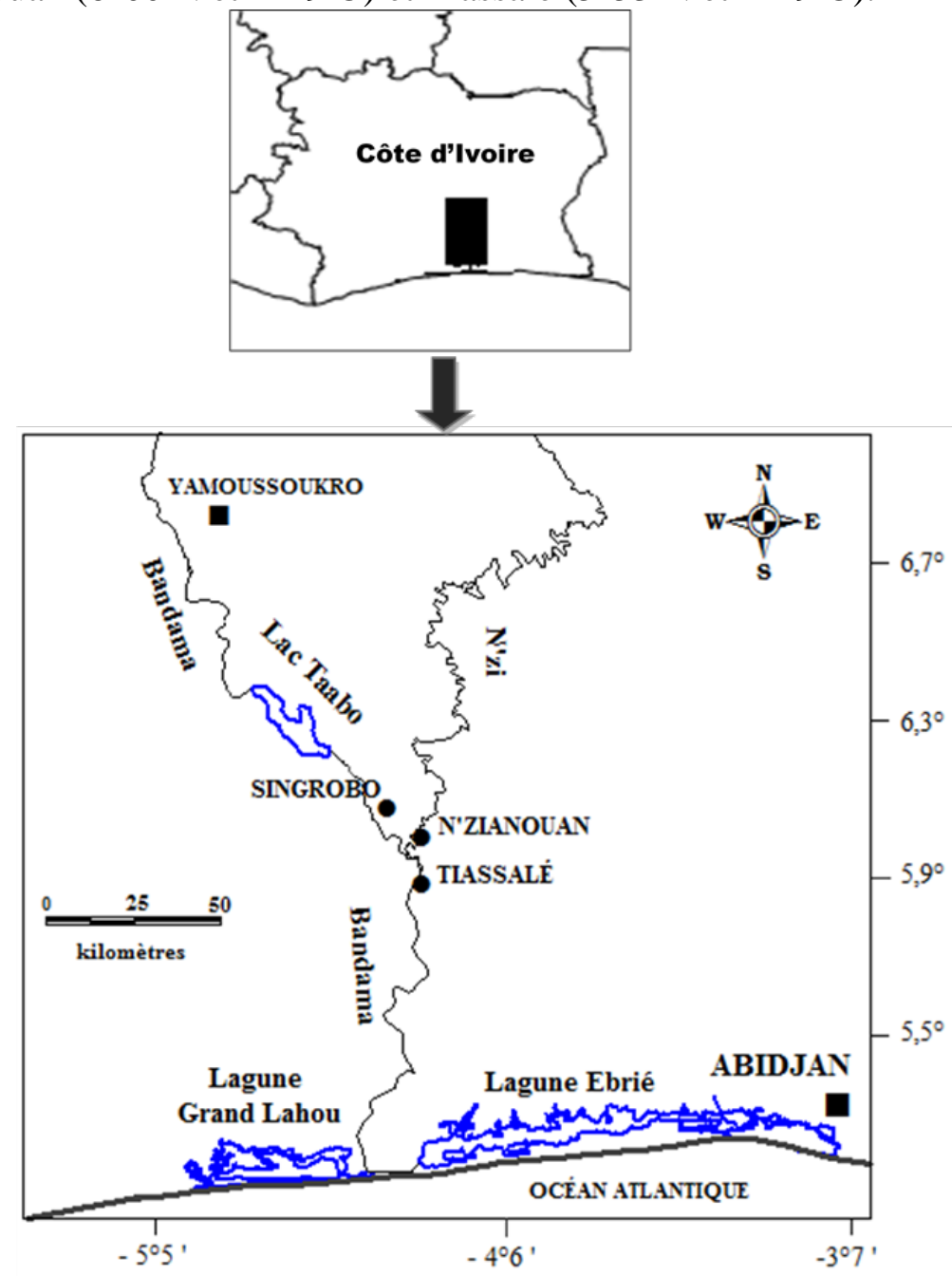

Figure 1 : Situation géographique des stations d'enquêtes et d'échantillonnage $(\bullet)$ sur le cours inférieur du fleuve Bandama 


\section{Echantillonnage des poissons}

Les poissons ont été collectés entre juillet 2019 et juin 2020 à partir des débarquements des pêcheurs locaux. Les enquêtes sur les débarquements de poissons se sont déroulées dans toutes les stations. Ces enquêtes ont duré 48 jours par station, à raison de 4 jours par mois pendant 12 mois. Pendant ces différentes missions, l'équipe a procédé à l'examen des captures journalières des pêcheurs. Les pêcheurs sont choisis au hasard et pour chaque débarquement, les informations suivantes ont été enregistrées:

- masse totale de la prise (kg);

- composition spécifique de la prise;

- masse et nombre d'individus par espèce.

Lorsque la capture est importante (plus de 5kg), un sous-échantillon choisi au hasard, constitué par des captures entières ou des fractions de capture a été analysé.

L'identification des poissons a été faite à l'aide de la clé dichotomique de Paugy et al. (2003a et b). Les spécimens ainsi échantillonnés ont été mesurés individuellement au mm près (longueurs standard [LS]) à l'aide d'un ichtyomètre et pesés au g près par une balance de précision $1 \mathrm{~g}$ de marque Teraillon.

\section{Traitement des données}

Un premier aspect des résultats a été présenté sous forme de liste et de pourcentage de dominance des espèces de poissons identifiés dans chaque localité en provenance de la pêche commerciale par Aboua et al. (2017).

Pour avoir une idée plus globale du peuplement de poissons du cours inférieur du fleuve Bandama, une analyse descriptive du peuplement ichtyologique a été réalisée à partir des indices biologiques et indicateurs de pression de pêche ci-dessous :

Richesse spécifique (S) et pourcentage d'occurrence (P.O)

La richesse spécifique totale ( $\mathrm{RS}=$ nombre d'espèces capturées) a été déterminée pour toute la période d'échantillonnage de juillet 2019 à juin 2020. Le pourcentage d'occurrence (P.O) a été calculé en faisant le pourcentage de mois où une espèce i est échantillonnée par rapport au nombre total de mois échantillonnés. Cette valeur est calculée selon la formule suivante :

$$
\text { P.O }=(\mathrm{Mi} / \mathrm{Mt}) \times 100
$$

$\mathrm{Mi}=$ nombre de mois où l'espèce $\mathrm{i}$ a été capturée;

Mt $=$ nombre total de mois prospectés.

La classification de Djakou et Thanon (1988) a été retenue pour la présente étude. Cette répartition s'établit comme suit :

80 à 100\% : Espèces très fréquentes ; 60 à 79\% : Espèces fréquentes ; 40 à $59 \%$ : Espèces assez fréquentes ; 20 à 39\% : Espèces accessoires et moins de $20 \%$ : Espèces accidentelles 


\section{Indice de diversité de Shannon ( $\left.H^{\prime}\right)$}

H' a été utilisé pour observer une évolution de la faune ichtyologique du milieu d'étude au cours du temps. Cet indice mesure également le degré d'organisation du peuplement (Amanieu et Lasserre, 1982). L'expression de cet indice est la suivante:

$$
\mathrm{H}^{\prime}=\sum(\mathrm{Ni} / \mathrm{N}) \times \log _{2}(\mathrm{Ni} / \mathrm{N})
$$

$\mathrm{Ni}$ : nombre d'individus d'une espèce donnée, i allant de 1 à $\mathrm{S}$ (nombre total d'espèces).

$\mathrm{N}$ : nombre total d'individus. H' est exprimé en unités d'information par individu ou bits/individu. H' est nul si l'échantillon est composé d'une seule espèce et maximal (de l'ordre de 5) si toutes les espèces de la communauté sont également représentées dans l'échantillon (Ludwig et Renolds, 1988).

Equitabilité (E)

L'équitabilité (E) permet d'étudier la répartition des abondances des espèces dans un écosystème (Hill, 1973). Ce paramètre a été estimé par la formule suivante:

$$
\mathrm{E}=\mathrm{H}^{\prime} / \log _{2} \mathrm{~S}
$$

Avec: $\mathrm{H}^{\prime}$ = indice de Shannon; $\mathrm{S}$ = richesse spécifique.

Elle varie de 0 à 1 . Elle est maximale quand les espèces ont des abondances identiques dans le peuplement. Elle est minimale quand une seule espèce domine tout le peuplement. Le programme GWBASIC a permis de calculer l'indice de Shannon et l'équitabilité.

Indice de similarité de Jaccard

L’indice de similarité de Jaccard (1908) a été utilisé dans le présent travail pour mesurer la similarité entre deux communautés ou stations. Cet indice est calculé selon la formule suivante:

$$
\mathrm{CJ}=\frac{\text { Nombre d'espèces communes aux deux communautés }}{\text { Nombre total d'espèces dans les deux communautés }} \times 100
$$

\section{Courbe $A B C$}

Dans le présent travail, l'hypothèse à vérifier est celle d'un milieu stressé. En effet, le nombre important de pêcheurs et la diversité d'engins de pêche déployés devraient entrainer un milieu stressé (Tah, 2012). Ainsi, la courbe ABC (Courbes de Comparaison Abondance/Biomasse) a été tracée à partir des données de la pêche commerciale. Les pourcentages cumulés d'abondance et de biomasse en fonction des rangs d'espèces dans le même repère donnent les courbes ABC. Selon (Warwick et Clarke, 1994 ; Attrill et Depledge, 1997 ; Kantoussan, 2007),

- Si la courbe des biomasses cumulées est au-dessus de celle des abondances alors le milieu est dans un état de non stress. 
- Si les courbes des biomasses et des abondances cumulées se confondent presque alors le milieu est dans une phase de stress léger.

- Si la courbe des biomasses cumulées est en dessous de celle de l'abondance alors le milieu est dans un état de stress important.

Tailles des spécimens des captures commerciales

Pour étudier l'impact de la pêche sur le peuplement de poissons du cours inférieur du fleuve Bandama, les espèces les plus fréquentes et abondantes dans les captures commerciales ont été considérées et regroupées comme suit pour chaque espèce : spécimens de tailles inférieures à la taille du plus petit individu mature ; spécimens de tailles comprises entre la taille du plus petit individu mature et celle de première maturité et enfin ceux de tailles supérieures à la taille de première maturité de l'espèce considérée. Les individus de la première tranche sont considérés comme des juvéniles, ceux de la deuxième tranche sont dits sub-adultes alors que ceux appartenant à la troisième catégorie sont dits adultes.

Traitements statistiques

L’ANOVA (1facteur) a été utilisée pour comparer les différentes moyennes observées sur les variables richesses spécifiques, indices de diversité et équitabilité. Le test du Khi-deux a permis de comparer les indices de similarité. Toutes les analyses statistiques ont été effectuées avec le logiciel Statsoft-Statistica, Inc. 7.0 et Excell 2003.

\section{Résultats}

\section{Répartition spatio-temporelle de l'ichtyofaune}

C'est un total de 44 espèces appartenant à 19 familles et 31 genres qui ont été recensées sur l'ensemble de la zone. La richesse spécifique varie d'un mois à un autre et d'un site à l'autre (Figures 2 et 3). Ainsi à Tiassalé, ce sont 39 espèces (88,88\% de l'ichtyofaune totale de la zone d'étude) pour 18 familles qui ont été recensées. Trente-sept (37) espèces (82,22\%) réparties entre 17 familles sont présentes à Singrobo alors que 33 espèces (73,33\%) appartiennent à 17 familles à N'Zianouan. À Tiassalé, le plus petit nombre d'espèces a été noté en décembre (7 espèces) et le plus grand nombre en mai (25 espèces). Concernant Singrobo, la richesse spécifique minimum obtenue est de 9 espèces en octobre alors que la maximale de 22 espèces est notée aux mois de novembre et mai. Les valeurs minimale et maximale de la richesse spécifique calculées à N'Zianouan sont respectivement 6 (décembre) et 21 (janvier). Les richesses spécifiques moyennes sont de $17 \pm 5$ espèces à Tiassalé, $16 \pm 4$ espèces à Singrobo et de $13 \pm 5$ espèces à N'Zianouan. Les différentes valeurs de richesse spécifique mensuelle dans chaque site, ont été soumises à l'ANOVA. Les résultats obtenus ( $p=0,104270 ; p>0,05)$ montre qu'il n'y a pas de différence significative entre les trois zones. 


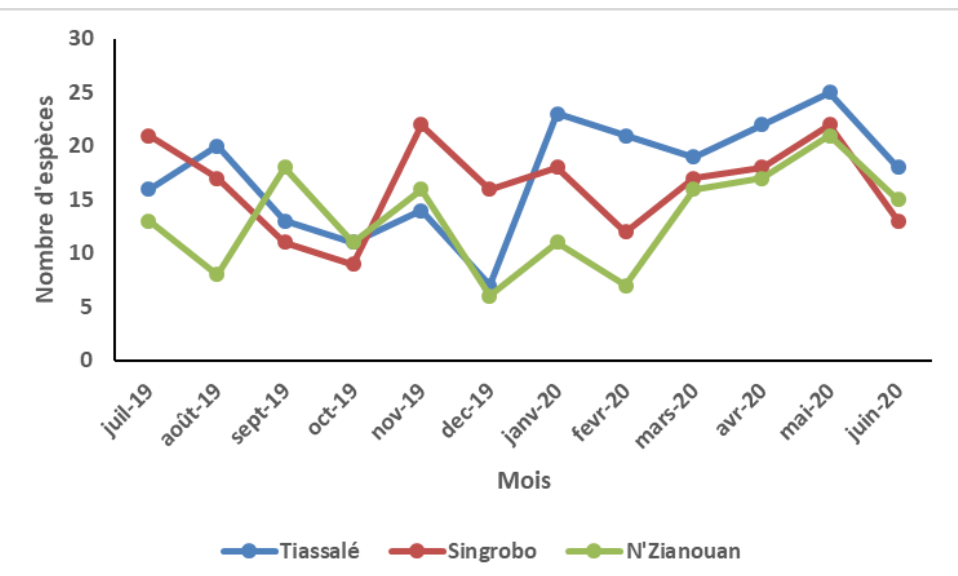

Figure 2 : Variation temporelle de la richesse spécifique à Tiassalé, Singrobo et N’Zianouan sur le cours inférieur du fleuve Bandama de juillet 2019 à juin 2020.

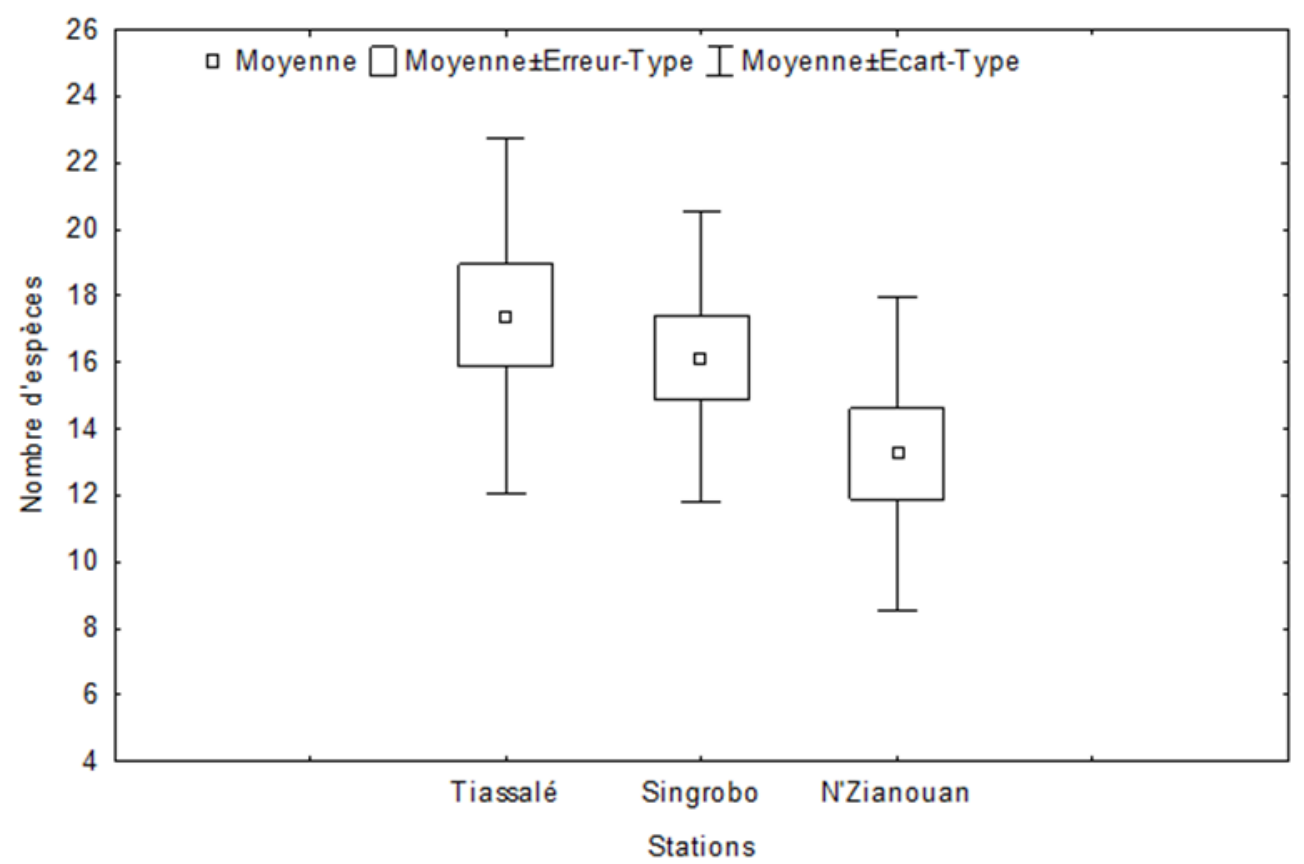

Figure 3 : Variation des moyennes de la richesse spécifique par station d'échantillonnage sur le cours inférieur du fleuve Bandama de juillet 2019 à juin 2020.

\section{Pourcentage d'occurrence}

L'analyse des occurrences mensuelles des espèces (Tableau 1) dans les captures de la pêche artisanale montre qu'à Tiassalé, 15\% des espèces sont très fréquentes dans la zone (Schilbe mandibularis, Brycinus macrolepidotus, Chrysichthys nigrodigitatus, Labeo coubie, Distichodus rostratus et Synodontis bastiani). Dans ce groupe, seule Chrysichthys nigrodigitatus a une occurrence mensuelle égale à $100 \%$. Les espèces Hemichromis fasciatus, 
Coptodon zillii, Mormyrops anguilloïdes et Papyrocranus afer qui font 12,5\% des espèces sont fréquentes dans les eaux de Tiassalé. Hydrocynus forskalii, Mormyrus rume, Barbus bynni occidentalis, Heterobranchus isopterus, Lates niloticus, Parachanna obscura, Labeo parvus, Trachinotus teraia et Synodontis schall, faisant $22,5 \%$ des espèces, sont considérées comme espèces assez fréquentes à Tiassalé.

Sept espèces (Heterobranchus longifilis, Oreochromis niloticus, Ctenopoma petherici, Sarotherodon galilaeus, Tylochromis jentinki, Clarias anguillaris et Awaous lateristriga) (17,5\% des espèces) apparaissent de façon accessoire dans la zone de Tiassalé. Les autres espèces $(32,5 \%)$ y viennent de façon accidentelle.

À Singrobo, Mormyrus rume, Chrysichthys nigrodigitatus, Synodontis bastiani, Marcusenius senegalensis (10,81\% des espèces) sont les espèces très fréquentes dans les captures. Hemichromis fasciatus, Coptodon zillii, Mormyrops anguilloïdes et Synodontis schall (10,81\% des espèces) sont fréquentes dans les prises. À Singrobo, les espèces Chrysichthys nigrodigitatus et Mormyrus rume présentent une fréquence de 100\% chacune. Un troisième groupe d'espèces constitué d'Oreochromis niloticus, Ctenopoma petherici, Sarotherodon galilaeus, Tylochromis jentinki, Parachanna obscura, Labeo coubie, Schilbe mandibularis, Brycinus macrolepidotus, Heterobranchus isopterus, Chromidotilapia guntheri, Auchenoglanis occidentalis et Barbus bynni waldroni (32,43\% des espèces) est considéré comme assez fréquent dans le milieu. Les espèces Barbus bynni occidentalis, Labeo parvus, Papyrocranus afer, Distichodus rostratus, Clarias anguillaris, Malapterurus electricus et Lates niloticus entrent de façon accessoire dans les captures. Les espèces restantes (27,03\% des espèces) entrent accidentellement dans les prises.

Dans les captures de N'Zianouan, seules les espèces Chrysichthys nigrodigitatus et Synodontis bastiani (6,06\% des espèces) sont très fréquentes dans les captures. L'espèce Chrysichthys nigrodigitatus a été échantillonnée tous les mois contrairement à Synodontis bastiani. L'ensemble des espèces Coptodon zillii, Mormyrus rume, Labeo coubie, Hemichromis fasciatus et Mormyrops anguilloïdes (15,15\% des espèces) est fréquemment capturé à N'Zianouan.

Tableau 1 : Distribution des occurrences mensuelles des espèces de poissons sur le cours inférieur du fleuve Bandama de juillet 2019 à juin 2020.

\begin{tabular}{ccccc}
\hline \multirow{2}{*}{ Familles } & Espèces & \multicolumn{3}{c}{$\%$ d'occurrence } \\
\cline { 3 - 5 } & & Tiassale & Singrobo & N'Zianouan \\
\hline Arapaimiidae & Heterotis niloticus & 8,33 & & \\
Notopteridae & Papyrocranus afer & 66,67 & 33,33 & 33,33 \\
Mormyridae & Mormyrops anguilloides & 66,67 & 75 & 66,67 \\
& Mormyrus rume & 75 & 100 & 66,67
\end{tabular}




\begin{tabular}{|c|c|c|c|c|}
\hline & Marcusenius senegalensis & 58,33 & 83,33 & 50 \\
\hline & Marcusenius ussheri & & 8,33 & \\
\hline \multirow[t]{5}{*}{ Alestidae } & Alestes baremoze & & 8,33 & \\
\hline & Brycinus nurse & 8,33 & 8,33 & 8,33 \\
\hline & Brycinus macrolepidotus & 83,33 & 41,67 & 41,67 \\
\hline & Brycinus imberi & & 8,33 & 8,33 \\
\hline & Hydrocynus forskalii & 41,67 & & 41,67 \\
\hline Distichodontidae & Distichodus rostratus & 83,33 & 41,67 & 41,67 \\
\hline \multirow[t]{4}{*}{ Cyprinidae } & Labeo parvus & 41,67 & 33,33 & 41,67 \\
\hline & Labeo coubie & 91,67 & 58,33 & 75 \\
\hline & Barbus bynni waldroni & 16,67 & 58,33 & 25 \\
\hline & Barbus bynni occidentalis & 41,67 & 25 & \\
\hline \multirow[t]{3}{*}{ Claroteidae } & Chrysichthys maurus & 8,33 & 8,33 & \\
\hline & Chrysichthys nigrodigitatus & 100 & 100 & 100 \\
\hline & Auchenoglanis occidentalis & 16,67 & 41,67 & 16,67 \\
\hline \multirow[t]{2}{*}{ Schilbeidae } & Schilbe intermidius & 8,33 & 8,33 & 16,67 \\
\hline & Schilbe mandibularis & 83,33 & 50 & 33,33 \\
\hline \multirow[t]{3}{*}{ Clariidae } & Clarias anguillaris & 33,33 & 33,33 & \\
\hline & Heterobranchus isopterus & 58,33 & 50 & 41,67 \\
\hline & Heterobranchus longifilis & 33,33 & & \\
\hline Malapteruridae & Malapterurus electricus & 16,67 & 33,33 & 25 \\
\hline \multirow[t]{3}{*}{ Mochokidae } & Synodontis bastiani & 83,33 & 91,67 & 83,33 \\
\hline & Synodontis schall & 41,67 & 66,67 & 33,33 \\
\hline & Synodontis punctifer & 8,33 & & \\
\hline Channidae & Parachanna obscura & 58,33 & 50 & 58,33 \\
\hline Latidae & Lates niloticus & 41,67 & 33,33 & 25 \\
\hline Carangidae & Trachinotus teraia & 50 & & 41,67 \\
\hline \multirow[t]{5}{*}{ Cichlidae } & Chromidotilapia guntheri & 16,67 & 41,67 & 8,33 \\
\hline & Hemichromis bimaculatus & & 16,67 & \\
\hline & Hemichromis fasciatus & 66,67 & 75 & 66,67 \\
\hline & Oreochromis niloticus & 25 & 41,67 & 41,67 \\
\hline & Sarotherodon galilaeus & 33,33 & 50 & 8,33 \\
\hline \multicolumn{5}{|l|}{ Tableau I suite } \\
\hline & Sarotherodon melanotheron & 16,67 & & 16,67 \\
\hline & Coptodon mariae & 8,33 & & \\
\hline & Coptodon zillii & 75 & 75 & 66,67 \\
\hline & Tylochromis jentinki & 33,33 & 50 & 25 \\
\hline Mugilidae & Liza falcipinnis & 8,33 & 8,33 & 16,67 \\
\hline Gobiidae & Awaous lateristriga & 25 & 16,67 & 25 \\
\hline Anabantidae & Ctenopoma petherici & 25 & 50 & 41,67 \\
\hline Mastacembelidae & Mastacembelus nigromarginatus & & 8,33 & \\
\hline Total & 44 & 39 & 37 & 33 \\
\hline
\end{tabular}


Les espèces Parachanna obscura, Marcusenius senegalensis, Brycinus macrolepidotus, Oreochromis niloticus, Labeo parvus, Trachinotus teraia, Ctenopoma petherici, Heterobranchus isopterus, Distichodus rostratus et Hydrocynus forskalii qui constituent 30,30\% des espèces sont assez fréquentes dans les captures.

On note que $24,24 \%$ des espèces composé de Lates niloticus, Papyrocranus afer, Schilbe mandibularis, Tylochromis jentinki, Awaous lateristriga, Synodontis schall, Malapterurus electricus et Barbus bynni waldroni sont accessoires dans les prises des pêcheurs. Les sept autres espèces (21,21\% des espèces) apparaissent accidentellement dans les captures.

\section{Indice de similarité}

Les indices de similarité de Jaccard (J) calculés entre les trois stations d'échantillonnage pour les 12 mois d'étude restent élevés. Le tableau 2 présente la similarité des familles et espèces entre Tiassalé, Singrobo et N'Zianouan. Les similarités sont plus élevées entre Tiassalé et N'Zianouan (94,44\% en familles et 78,05\% en espèces) et entre Singrobo et N'Zianouan (88,88\% en familles et $75 \%$ en espèces). Les plus faibles similarités $(84,21 \%$ en familles et $71,11 \%$ en espèces) sont observées entre Tiassalé et Singrobo. Il n'existe pas de différence significative $\left(\chi^{2}=0,12 ; p=0,07\right)$ entre les indices de similarité. Néanmoins, ces valeurs montrent une démarcation des deux stations Tiassalé et N'Zianouan de Singrobo.

\section{Diversité}

Les indices de diversité (H’) (Figure 4) et l'équitabilité (E) (Figure 5) présentent des variations mensuelles. Les valeurs de l'équitabilité varient très peu d'un mois à l'autre sur le cours inférieur du fleuve Bandama. Les plus fortes valeurs ( $\mathrm{H}^{\prime}=2,58$ en mai ; $\mathrm{E}=0,88$ en juillet) et les plus faibles $\left(\mathrm{H}^{\prime}=\right.$ 1,56 en décembre; $E=0,79$ en juin et octobre) donnent les moyennes mensuelles suivantes : 2,23 $\pm 0,37$ (H') et $0,84 \pm 0,04$ (E).

À Tiassalé, un premier pic de H' $(2,54)$ est obtenu en août, plus faible que le deuxième $(2,67)$ observé en janvier. Un troisième plus élevé $(2,70)$ est noté en mai. La valeur la plus faible $\left(\mathrm{H}^{\prime}=1,34\right)$ est obtenue en décembre. La plus grande valeur de l'équitabilité $(E=0,88)$ est observée en juillet et la plus faible en juin $(E=0,78)$. Les indices moyens mensuels dans cette localité sont de 2,34 $\pm 0,4\left(\mathrm{H}^{\prime}\right)$ et de $0,85 \pm 0,03(\mathrm{E})$.

Concernant Singrobo, le pic de H' est obtenu en juillet $(2,60)$ alors que la plus faible valeur est obtenue en octobre $\left(\mathrm{H}^{\prime}=1,74\right)$. La valeur de l'équitabilité est plus élevée en août $(E=0,90)$. La plus faible valeur est celle de novembre $(E=0,78)$. Les valeurs moyennes de $H^{\prime}$ et de $E$ sont respectivement de $2,27 \pm 0,28 /$ mois et de $0,83 \pm 0,04 /$ mois. 
Au niveau de N'Zianouan, on note des valeurs de H' plus élevées en septembre $(2,61)$ et plus faible en décembre $(1,28)$ avec une moyenne mensuelle de $2,09 \pm 0,43$. Concernant l'equitabilité, la plus forte valeur $(0,92)$ est obtenue en décembre. Le mois d'octobre présente la plus faible valeur $(0,77)$. La valeur moyenne de $\mathrm{E}$ est de $0,84 \pm 0,05 /$ mois.

Tableau 2 : Richesse et similarité taxonomiques comparées des stations de Tiassalé, Singrobo et N'Zianouan sur le cours inférieur du fleuve Bandama de juillet 2019 à juin 2020. ( $\mathrm{J}=$ Indice de diversité de Jaccard)

\begin{tabular}{|c|c|c|c|c|c|}
\hline Taxons & Localités & Richesse & $\begin{array}{c}\text { Taxons } \\
\text { communs }\end{array}$ & $\begin{array}{c}\text { Taxons } \\
\text { non communs }\end{array}$ & J (\%) \\
\hline \multirow[t]{2}{*}{ Familles } & Tiassalé & 18 & \multirow{2}{*}{16} & 2 & \multirow{2}{*}{84,21} \\
\hline & Singrobo & 17 & & 1 & \\
\hline \multirow[t]{2}{*}{ Espèces } & Tiassalé & 40 & \multirow{2}{*}{32} & 8 & \multirow{2}{*}{71,11} \\
\hline & Singrobo & 37 & & 5 & \\
\hline \multirow[t]{2}{*}{ Familles } & Tiassalé & 18 & \multirow{2}{*}{17} & 1 & \multirow{2}{*}{94,44} \\
\hline & N'Zianouan & 17 & & 0 & \\
\hline \multirow[t]{2}{*}{ Espèces } & Tiassalé & 40 & \multirow{2}{*}{32} & 8 & \multirow{2}{*}{78,05} \\
\hline & N'Zianouan & 33 & & 1 & \\
\hline \multirow[t]{2}{*}{ Familles } & Singrobo & 17 & \multirow{2}{*}{16} & 1 & \multirow{2}{*}{88,88} \\
\hline & N'Zianouan & 17 & & 1 & \\
\hline \multirow[t]{2}{*}{ Espèces } & Singrobo & 37 & \multirow{2}{*}{30} & 7 & \multirow{2}{*}{75} \\
\hline & N'Zianouan & 33 & & 3 & \\
\hline
\end{tabular}




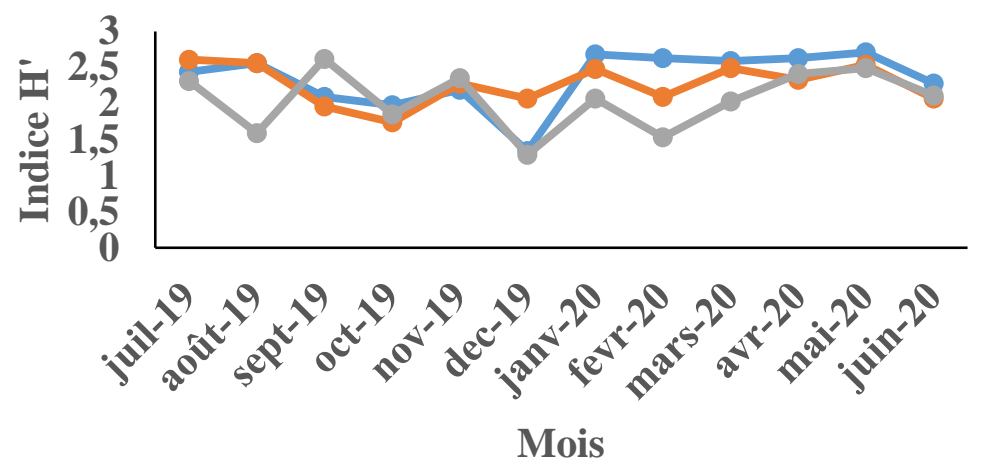

-Tiassalé - Singrobo - - N'Zianouan

Figure 4 : Variations mensuelles des indices de Shannon calculés à Tiassalé, Singrobo et N’Zianouan sur le cours inférieur du fleuve Bandama de juillet 2019 à juin 2020.

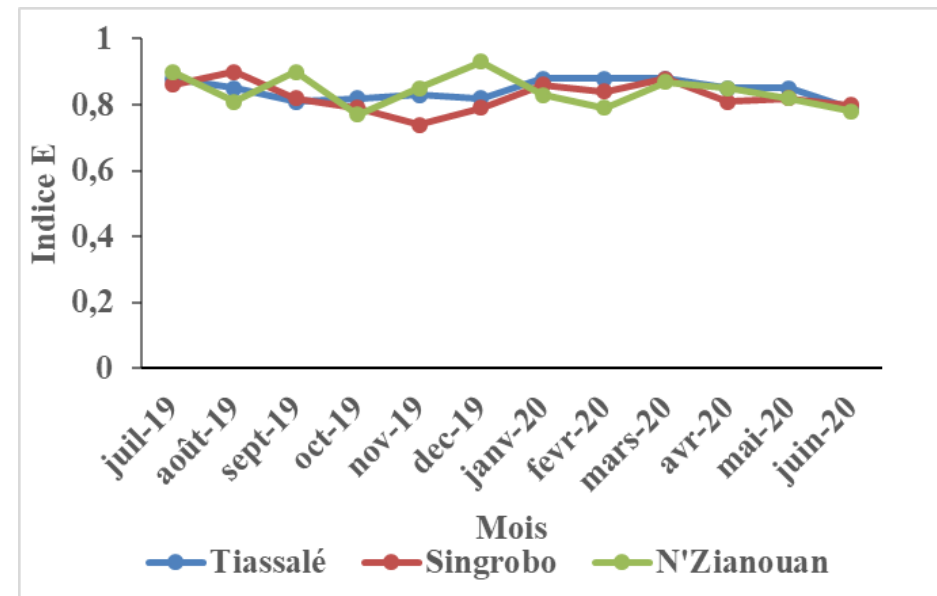

Figure 5 : Variations mensuelles des indices d'équitabilité calculés à Tiassalé, Singrobo et N’Zianouan sur le cours inférieur du fleuve Bandama de juillet 2019 à juin 2020.

Les différentes valeurs des indices $\mathrm{H}^{\prime}$ et $\mathrm{E}$ soumises à l'ANOVA montrent qu'il n'y a pas de différence significative $(p>0,05)$ entre les trois stations d'étude avec $p=0,155567$ pour H' et $p=0,515174$ pour E.

\section{Evaluation du stress écologique sur le cours inférieur du fleuve Bandama}

La distribution comparée des biomasses et des abondances dans les localités de Tiassalé, Singrobo et N'Zianouan est illustrée par la figure 6 . À Tiassalé, la distribution des biomasses et celle de l'abondance sont presque confondues (Graphe A). Dans les stations de Singrobo et de N'Zianouan, l'écart est beaucoup plus marqué avec la distribution des biomasses au-dessus de celle des abondances (Graphe B et C). 

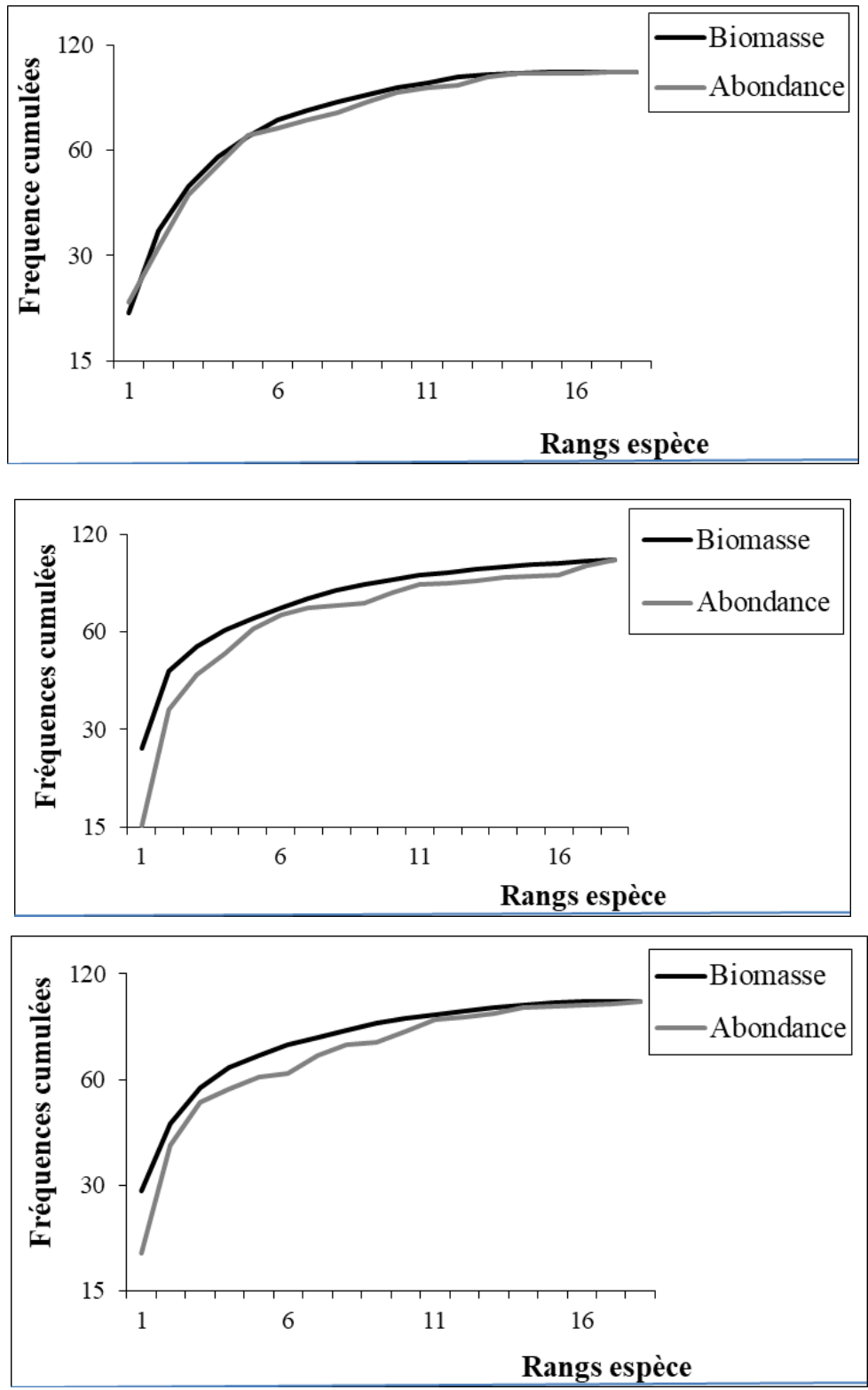

Figure 6 : Comparaison des distributions des abondances et biomasses dans les captures commerciales de poisson à Tiassalé (A), Singrobo (B) et N'Zianouan (C) sur le cours inférieur du fleuve Bandama de juillet 2019 à juin 2020. (Courbes ABC)

\section{Fréquence des tailles des spécimens}

Les résultats de variation de tailles des spécimens montrent que dans la localité de Tiassalé, le groupe constitué de Brycinus macrolepidotus, Clarias anguillaris, Tylochromis jentinki, Heterobranchus isopterus, Schilbe 
mandibularis, Labeo parvus et Chrysichthys maurus faisant $21,21 \%$ des espèces considérées a été majoritairement capturé au stade adulte (tableau 3). Un deuxième groupe constitué d'Auchenoglanis occidentalis, Hemichromis fasciatus, Trachinotus teraia, Marcusenius senegalensis, Mormyrops anguilloides, Brycinus nurse, Hydrocynus forskalii (21,21\% des espèces considérées) présentent majoritairement une taille d'individus sub-adultes au cours de leur capture. Les autres espèces (57,58\% des espèces considérées) ont été capturées avec des jeunes individus.

Le tableau 4 montre la distribution des tailles à Singrobo. Les espèces Lates niloticus, Tylochromis jentinki, Brycinus macrolepidotus et Labeo parvus représentent 14,29\% des espèces considérées. Elles sont caractérisées par des individus adultes. Les espèces Hemichromis fasciatus, Coptodon zillii, Mormyrops anguilloides, Brycinus imberi, Brycinus nurse, Heterobranchus isopterus, Schilbe mandibularis, Auchenoglanis occidentalis et Parachanna obscura (32,14\% des espèces considérées) présentent plus d'individus subadultes dans les captures. Les autres individus capturés appartenant aux autres espèces considérées $(53,57 \%)$ sont majoritairement des juvéniles.

Dans les débarcadères de N'Zianouan (Tableau 5), Labeo parvus, Brycinus macrolepidotus, Lates niloticus, Schilbe mandibularis et Labeo coubie (16,13\% des espèces considérées) sont des espèces présentant des individus généralement adultes. Le groupe composé de Brycinus imberi, Brycinus nurse, Heterobranchus isopterus, Hemichromis fasciatus, Schilbe mandibularis, Hydrocynus forskalii, Coptodon zillii, Tylochromis jentinki et Parachanna obscura (29,03\% des espèces considérées) présente des captures essentiellement constituées d'individus sub-adultes. Le dernier groupe (54,84\% des espèces considérées) est constitué essentiellement de juvéniles.

Tableau 3 : Répartition selon la maturité des principales espèces de poissons capturés à la station de Tiassalé.

\begin{tabular}{|c|c|c|c|c|}
\hline Espèces & Nombre & Juvénile (\%) & Sub-adulte (\%) & Adulte (\%) \\
\hline Papyrocranus afer & 95 & 94,74 & 5,26 & \\
Mormyrops anguilloides & 125 & & 68 & 32 \\
Mormyrus rume & 158 & 82,28 & 7,59 & 10,13 \\
Marcusenius senegalensis & 230 & 42,5 & 55,33 & 2,17 \\
Brycinus nurse & 5 & & 100 & 45 \\
Brycinus macrolepidotus & 400 & 32,5 & 22,5 & 7,35 \\
Hydrocynus forskalii & 68 & & 92,65 & 7,25 \\
Distichodus rostratus & 359 & 80,87 & 11,88 & 100 \\
Labeo parvus & 181 & & & 33,85 \\
Labeo coubie & 655 & 52,92 & 13,23 &
\end{tabular}




\begin{tabular}{|c|c|c|c|c|}
\hline Chrysichthys maurus & 15 & & & 100 \\
\hline Chrysichthys nigrodigitatus & 905 & 67,51 & 31,27 & 1,22 \\
\hline Auchenoglanis occidentalis & 10 & & 100 & \\
\hline Schilbe intermedius & 18 & & 100 & \\
\hline Schilbe mandibularis & 230 & 2,17 & 45,65 & 52,17 \\
\hline Clarias anguillaris & 42 & & 11,9 & 88,1 \\
\hline Heterobranchus isopterus & 125 & 16 & 36 & 48 \\
\hline Synodontis bastiani & 528 & 94,7 & 2,27 & 3,03 \\
\hline Synodontis schall & 500 & 72,2 & 26,8 & 1 \\
\hline Parachanna obscura & 55 & 54,55 & 27,27 & 18,18 \\
\hline Lates niloticus & 41 & 75,61 & & 24,39 \\
\hline Trachinotus teraia & 150 & 100 & & \\
\hline Chromidotilapia guntheri & 26 & 76,92 & 23,08 & \\
\hline Hemichromis fasciatus & 195 & & 97,44 & 2,56 \\
\hline Oreochromis niloticus & 27 & 70,83 & 29,17 & \\
\hline Sarotherodon galilaeus & 60 & 70 & 30 & \\
\hline Sarotherodon melanotheron & 108 & 95,37 & & 6,63 \\
\hline Coptodon mariae & 10 & & 100 & \\
\hline Coptodon zillii & 395 & 49,37 & 39,24 & 11,39 \\
\hline Tylochromis jentinki & 62 & & 33,87 & 66,13 \\
\hline Liza falcipinnis & 53 & 77,36 & 22,64 & \\
\hline Ctenopoma petherici & 80 & 78,82 & 21,18 & \\
\hline
\end{tabular}

Tableau 4 : Répartition selon la maturité des principales espèces de poissons capturés à la station de Singrobo.

\begin{tabular}{|c|c|c|c|c|}
\hline Espèces & Nombre & Juvénile (\%) & Sub-adulte (\%) & Adulte (\%) \\
\hline Papyrocranus afer & 29 & 100 & & \\
Mormyrops anguilloides & 131 & 13,64 & 45,45 & 40,91 \\
Mormyrus rume & 305 & 86,89 & 13,11 & \\
Marcusenius senegalensis & 395 & 95,92 & 4,08 & \\
Brycinus nurse & 9 & & 100 & \\
Brycinus macrolepidotus & 40 & & 33,33 & 106,67 \\
Brycinus imberi & 7 & & 100 & \\
Distichodus rostratus & 66 & 88,88 & 11,12 & \\
Labeo parvus & 230 & & 18,75 & \\
Labeo coubie & 211 & 50 & &
\end{tabular}




\begin{tabular}{|c|c|c|c|c|} 
Barbus bynni waldroni & 65 & 92,31 & 7,69 & 0,98 \\
Chrysichthys nigrodigitatus & 815 & 88,96 & 10,06 & 100 \\
Auchenoglanis occidentalis & 45 & & 74,15 & 16,1 \\
Schilbe mandibularis & 205 & 9,75 & 10,81 & 18,92 \\
Clarias anguillaris & 37 & 70,27 & 88,69 & \\
Heterobranchus isopterus & 53 & 11,31 & 7,47 & 12,31 \\
Synodontis bastiani & 415 & 92,53 & 2,43 & 100 \\
Synodontis schall & 411 & 97,57 & 50,77 & 5,46 \\
Parachanna obscura & 65 & 36,92 & & 7,88 \\
Lates niloticus & 20 & & 18,18 & 18,18 \\
Chromidotilapia guntheri & 165 & 76,36 & 92,12 & 4,54 \\
Hemichromis fasciatus & 241 & & 27,27 & 24,37 \\
Oreochromis niloticus & 44 & 54,55 & 4,54 & 75,38 \\
Sarotherodon galilaeus & 66 & 90,92 & 75,63 & \\
Coptodon zillii & 636 & & 24,62 & \\
Tylochromis jentinki & 65 & 100 & & \\
Liza falcipinnis & 14 & 92 & & \\
Ctenopoma petherici & 125 & & & \\
\hline
\end{tabular}

Tableau 5 : Répartition selon la maturité des principales espèces de poissons capturés à la station de N'Zianouan.

\begin{tabular}{|c|c|c|c|c|}
\hline Espèces & Nombre & Juvénile (\%) & Sub-adulte (\%) & Adulte (\%) \\
\hline Papyrocranus afer & 38 & 100 & & \\
Mormyrops anguilloïdes & 90 & 55,56 & 40 & 4,44 \\
Mormyrus rumé & 147 & 89,8 & 10,2 & \\
Marcusenius senegalensis & 160 & 70 & 30 & 55,33 \\
Brycinus nurse & 8 & & 100 & 15,62 \\
Brycinus macrolepidotus & 150 & & 44,67 & \\
Brycinus imberi & 32 & & 84,38 & 100 \\
Hydrocynus forskalii & 50 & & 12,26 & 75 \\
Distichodus rostratus & 212 & 81,6 & 25 & 50 \\
Labeo parvus & 180 & 50 & &
\end{tabular}




\begin{tabular}{|c|c|c|c|c|} 
Barbus bynni waldroni & 21 & 100 & & \\
Chrysichthys nigrodigitatus & 626 & 93,61 & 3,83 & 2,56 \\
Schilbe mandibularis & 68 & & 50 & 50 \\
Heterobranchus isopterus & 62 & 19,35 & 64,52 & 16,13 \\
Synodontis bastiani & 401 & 80,05 & 19,95 & 6,06 \\
Synodontis schall & 231 & 80,52 & 13,42 & 24,52 \\
Parachanna obscura & 53 & 37,74 & 37,74 & 78,26 \\
Lates niloticus & 23 & 17,39 & 4,35 & 10,81 \\
Trachinotus teraia & 37 & 83,78 & 5,41 & 2,86 \\
Chromidotilapia guntheri & 15 & 100 & & 12 \\
Hemichromis fasciatus & 175 & 22,85 & 74,29 & 12 \\
Oreochromis niloticus & 50 & 76 & 25 & 4,5 \\
Sarotherodon galilaeus & 16 & 75 & & 7,79 \\
Sarotherodon melanotheron & 111 & 95,5 & 88,39 & 3,33 \\
Coptodon zillii & 603 & 3,82 & 66,67 & \\
Tylochromis jentinki & 33 & & 10,28 & \\
Ctenopoma petherici & 214 & 89,72 & & \\
\hline
\end{tabular}

\section{Discussion}

\section{Variation de la faune ichtyologique et des indices biologiques}

La présente richesse spécifique observée dans les captures sur le cours inférieur du fleuve Bandama inclut l'essentiel des familles de poisson de l'ensemble du bassin du fleuve Bandama. L'organisation spatio-temporelle de la faune piscicole du cours inférieur du fleuve Bandama est caractérisée par une faible variabilité. Cette observation s'est traduite par des variations non significatives de la richesse spécifique et des indices de diversité et d'équitabilité entre les trois zones d'échantillonnage. La relative homogénéité de la communauté piscicole serait en rapport avec l'échelle spatiale relativement réduite que constitue la zone d'étude. En effet, les paramètres physico-chimiques des eaux, sont quasi-similaires dans les zones prospectées (Boguhé, 2015). Cela permet vraisemblablement aux poissons de coloniser tous les biotopes du cours inférieur du fleuve Bandama par des migrations d'amplitude plus ou moins grande.

\section{Evaluation du stress écologique}

Concernant l'évaluation du stress écologique, la méthode ABC semble traduire efficacement les changements dans la structure des peuplements exploités (Blanchard et al., 2004). La méthode ABC appliquée au peuplement 
de Tiassalé, indique que les courbes d'abondance et de biomasse sont proches de la phase de stress léger dans la localité de Tiassalé. Ces observations montrent que ce milieu peut être considéré comme un écosystème modérément stressé, selon le modèle théorique de Warwick (1986).

Selon Jennings et Kaiser (1998) ; Jennings et al. (1998), dans les milieux aquatiques, l'exploitation halieutique s'exerce d'abord sur des espèces de stratégie-k. Dans ce cas, la proportion d'espèces de grande taille diminue logiquement. À l'opposé, les espèces de petite taille faiblement exploitées et libérées de la prédation se développent (Rice et Gislason, 1996; Daan et al., 2005). Le peuplement se trouve alors dominé par des espèces de petite taille. Dans la localité de Tiassalé, les impacts de l'exploitation halieutique semblent avoir atteint ce niveau de modification du peuplement.

Dans les localités de Singrobo et de N'Zianouan, la courbe de biomasse est au-dessus de celle de l'abondance. Cela traduirait des milieux non stressés. La pression de pêche sur les espèces de stratégie-k serait ainsi soutenable. La forte présence dans les captures d'espèces naturellement à fortes biomasse (Labeo coubie, Distichodus rostratus, Heterotis niloticus) pourrait également expliquer cette situation. Une autre raison serait le comportement de certains pêcheurs qui ont tendance à jeter les plus petits individus capturés de peur de subir la loi. Néanmoins, l'écart moins important entre ces deux courbes traduit des situations qui pourraient tendre vers un état de stress si aucune mesure de protection n'est appliquée dans ces deux localités. Dans ces conditions, l'étude de la gamme de taille des individus capturés traduit mieux les effets de la pêche que la méthode $A B C$.

\section{Examen des tailles des spécimens}

La présente étude montre qu'un peu plus de la moitié des espèces capturées à Tiassalé (57,58\%), Singrobo (53,57\%) et N'Zianouan (54,84\%) sont des juvéniles. Ce constat augure d'une pression de pêche élevée et finalement presqu'identique dans l'ensemble des trois localités du cours inférieur du fleuve Bandama. La présence remarquée des juvéniles dans les captures montre que le faible maillage des engins de pêche (10 mm ; $20 \mathrm{~mm})$ utilisé dans le cours inférieur du Bandama est préjudiciable aux juvéniles. Cette situation devrait encourager les gouvernants dans leur prise de décision pour la protection de la ressource. Une telle situation a conduit Ouattara et al. (2006) à proposer la fermeture de la pêche sur le lac d'Ayamé pendant la saison de reproduction des poissons. Cela permettrait d'éviter le recrutement élevé de spécimens de petite taille nés avec la montée des eaux. L'application d'une telle décision sur le cours inférieur du fleuve Bandama associée à un contrôle strict des mailles des engins s'avère plus que nécessaire pour une gestion durable des ressources halieutiques. 


\section{Conclusion}

Le présent travail est une contribution à la gestion responsable des ressources exploitées dans la pêcherie du cours inférieur du fleuve Bandama. Au terme de cette étude, quelques points sont à retenir.

En termes de composition des captures, 44 espèces appartenant à 19 familles et 31 genres composent la faune ichtyologique exploitée sur le cours inférieur du fleuve Bandama entre juillet 2019 et juin 2020. Parmi ces espèces, 6 sont plus constantes dans les débarquements des pêcheurs. Il s’agit de Chrysichthys nigrodigitatus, Tilapia zillii, Synodontis bastiani, Synodontis schall et Marcusenius senegalensis.

La zone de Tiassalé qui présente la plus grande richesse spécifique est la plus productrice. L'essentiel de la production est fourni par les Cichlidae. L'analyse du stress écologique montre des zones de pêche proches d'un état de stress qui pourrait s’aggraver si aucune mesure de gestion n'est envisagée. Cette mesure est d'autant plus importante que plus de la moitié des individus de poisson capturés est juvénile.

\section{References:}

1. Lalèyè P., Chikou A., Philippart J-C., Teugels G., Vandewalle P. (2004). Etude de la diversité ichtyologique du bassin du fleuve Ouémé au Bénin (Afrique de l’Ouest). Cybium, 28 (4): 329-339.

2. Montchowui E., Chikou A., Kogbeto M.J., Lalèyè P. (2008). Biodiversity and structure of fish communities in Lake Hlan, Benin. International Journal of Biology and Chemical Sciences, 2 (2):196206.

3. Wu J., Wang J., He Y., Cao W. (2011). Fish assemblage structure in the Chishui River, a protected tributary of the Yangtze River. Knowledge and Management of aquatic Ecosystems, 400 (11):1-14.

4. Kamelan T.M., Yao S.S., Kouamé K.A., N’Zi K.G., Kouamélan E.P. (2013). Ichthyofauna of the Dodo River (Côte d'Ivoire, West Africa): update and influence of environmental variables on the distribution of species. Journal of Applied Biosciences, 71 (1):57735785.

5. Kantoussan J. (2007). Impact de la pression de pêche sur l'organisation des peuplements de poissons: Applications aux retenues artificielles de Sélingué et de Manantali, Mali Afrique de l’Ouest. Thèse de Doctorat, Mention halieutique Agrocampus de Renne, France, 195p.

6. Da Costa K.S., Konan K.F. (2005). Lac Kossou: Potentiel halieutique et modalité d'un développement durable de la pêche. FAO/PMEDP, Projet Pilote Pêche Kossou GCP/INT/735/UK, Rapport. Expertise, 200p. 
7. Da Costa K.S., Dietoa Y.M. (2007). Typologie de la pêche sur le lac Faé (Côte d'Ivoire) et implication pour une gestion rationnelle des ressources halieutiques. Bulletin Français pêche et pisciculture, 384: 1-14.

8. Tah L., Da Costa K.S., Kouassi N.J., Moreau J. (2009). Preliminary assessment of major Tilapiine fishes exploited by artisanal gillnet fishery in Lake Ayamé I (Bia basin, Côte d'Ivoire). Journal of Fisheries International, 4 (4): 83-90.

9. Koudou D.A. (2012). La pêche sur le lac de Taabo. Thèse de Doctorat, Université Félix Houphouët-Boigny, Abidjan, Côte d'Ivoire, 389p.

10. Aboua R.D.B. (2012). Développement d'un indice d'intégrité biotique piscicole pour la préservation de la biodiversité du fleuve Bandama. Thèse de Doctorat, Université Félix Houphouët-Boigny, Abidjan, Côte d'Ivoire, 227p.

11. Paugy D., Lévêque C., Teugels G.G. (éds). (2003a). Faune des poissons d'eaux douces et saumâtres de l'Afrique de l'Ouest. Tome 1. IRD (Paris), MNHN (Paris), MRAC (Tervuren), 457p.

12. Paugy D., Lévêque C., Teugels G.G. (éds). (2003b). Faune des poissons d'eaux douces et saumâtres de l'Afrique de l'Ouest. Tome 2. IRD (Paris), MNHN (Paris), MRAC (Tervuren), 815p.

13. Aboua, B.R.D., Kien K. B., Berte S., Kouamelan E. P. (2017). Fish species, catch per unit effort (cpue) and size structure in the lower Bandama river (Côte d'Ivoire, West Africa). International journal of biosciences, 10(4): 102-112.

14. Djakou R., Thanon S.Y. (1988). Écologie Afrique intertropicale. Édition Bordas, Paris, 191p.

15. Amanieu M., Lasserre G. (1982). Organisation et évolution des peuplements lagunaires. Oceanologica Acta, pp 201-213.

16. Ludwig J.A., Renolds J.F. (1988). Statistical ecology: A primer on methods and computing. John Wiley and Sons, New York, 7p.

17. Hill M.O. (1973). Diversity and evenness: a unifying notation and its consequences. Ecology, 54: 427-432.

18. Jaccard P. (1908). Nouvelles recherches sur la distribution florale. Bulletin de la Société Vaudoise des Sciences Naturelles, 44: 223-270.

19. Tah L. (2012). Exploitation du lac d'Ayamé I (Côte d'Ivoire) après le départ des pêcheurs «bozo» en 1998 et impact sur les structures démographiques des populations ichtyologiques. Thèse de Doctorat, Université Félix Houphouët-Boigny, Abidjan, Côte d'Ivoire, 177p.

20. Warwick R.M., Clarke K.R. (1994). Relearning the AB: taxonomic changes and abundance/ biomass relationships in disturbed benthic communities. Marine Biology, 118: 739-744. 
21. Attrill M.J., Depledge M.H. (1997). Community and population indicators of ecosystem health: target inglinks between levels of biological organization. Aquatic Toxicology, 38: 183-197.

22. Boguhé G.F.D.H. (2015). Biologie de la reproduction et exploitation de deux espèces de crevette du genre Macrobrachium: $M$. macrobrachion (Herklots, 1851) et M. vollenhovenii (Herklots, 1857) du fleuve Bandama (Côte d'Ivoire). Thèse de Doctorat, Université Félix Houphouët-Boigny, Abidjan, Côte d'Ivoire, 206p.

23. Blanchard F., LeLoc'h F., Hily C., Boucher J. (2004). Fishing effects on diversity, size and community structure of the benthic invertebrate and fish mega fauna on the Bay of Biscay coast of France. Marine Ecology Progress Series, 280: 249-260.

24. Warwick R.M. (1986). A new method for detecting pollution effects on marine macrobenthic communities. Marine Biology, 92: 557-562.

25. Jennings S., Kaiser M.J. (1998). The effects of fishing on marine ecosystems. Advances in Marine Biology, 34: 201-352.

26. Jennings S., Reynolds J.D., Mills S.C. (1998). Life history correlates of responses to fisheries exploitation, Proceedings of Royal Society. Biology Sciences, 265: 333-339.

27. Rice J., Gislason H. (1996). Patterns of change in the size spectra of numbers and diversity of the North Sea fish assemblage, as reflected in surveys and models. ICES Journal of Marine Science, 53: 12141225.

28. Daan N., Gislason H., Pope J.G., Rice J.C. (2005). Changes in the North Sea fish community: evidence of indirect effects of fishing? ICES Journal of Marine Science, 62: 177-188.

29. Ouattara M., Gourène G., Vanga A.F. (2006). Propositions de fermeture saisonnière de la pêche en vue d'une exploitation durable du poisson au lac d'Ayamé (Côte d'ivoire). Tropicultura, 24 (1): 7-13. 